\section{CENTRO DE CIÊNCIAE TECNOLOGIATÊXTIL}

\author{
www.2c2t.uminho.pt
}

\title{
Size and aging effects on antimicrobial efficiency of silver nanoparticles coated on polyamide fabrics activated by atmospheric DBD plasma
}

Andrea Zille $^{* 1}$, Margarida M. Fernandes², Antonio Francesko², Tzanko Tzanov², Marta Fernandes ${ }^{1}$, Fernando Ribeiro Oliveira ${ }^{3}$, Luís Almeida1 ${ }^{1}$, Teresa Amorim ${ }^{1}$, Noémia Carneiro ${ }^{1}$, Maria Fátima Esteves ${ }^{1}$ and António Pedro Souto ${ }^{1}$

\section{2C2T, University of Minho. *Email: azille@2c2t.uminho.pt}

2 Group of Molecular and Industrial Biotechnology, Department of Chemical Engineering, Universitat Politecnica de Catalunya, Terrassa, Spain.

3 Departamento de Engenharia Têxtil, Universidade Federal do Rio Grande do Norte UFRN, Natal, Brazil.

\section{Introduction}

Recently, renewed interest has arisen in silver nanoparticles for biomedical devices because of their high surface energy, enhanced physicochemical and biological properties and extremely large surface area, which provides better contact with microorganisms. Atmospheric plasma is an alternative and costcompetitive method to wet chemical nanoparticles deposition methods, avoiding the need of toxic solvents, expensive vacuum equipment and allowing continuous and uniform processing of material surfaces. However, there are no reports on the size and time-dependent antimicrobial, physical and chemical surface effects of the silver nanoparticles immobilized on plasma functionalized polymers. Thus, the purposes of this study were: (i) the silver nanoparticle size and aging effects after 30 days on the antimicrobial activity after deposition onto DBD plasma-treated polyamide 6,6 fabrics, and (ii) the aging effect on the physico-chemical binding mechanism between different sized silver nanoparticles and the plasma treaded polyamide 6,6. Five different in size commercial silver nanoparticles have been employed (10, 20, 4060 and 100 $\mathrm{nm})$.

\section{XPS analysis}

Deconvolution of the C1s and 01s core levels after plasma treatment show new peaks attributed both to the huge amount of newly formed polar groups and to the silver nanoparticles interacting to the fabric via hydroxyl and carboxylic groups as confirmed by the size-dependent positive shift in binding energy of $\mathrm{Ag}$ $3 \mathrm{~d} 5 / 2$ peaks.
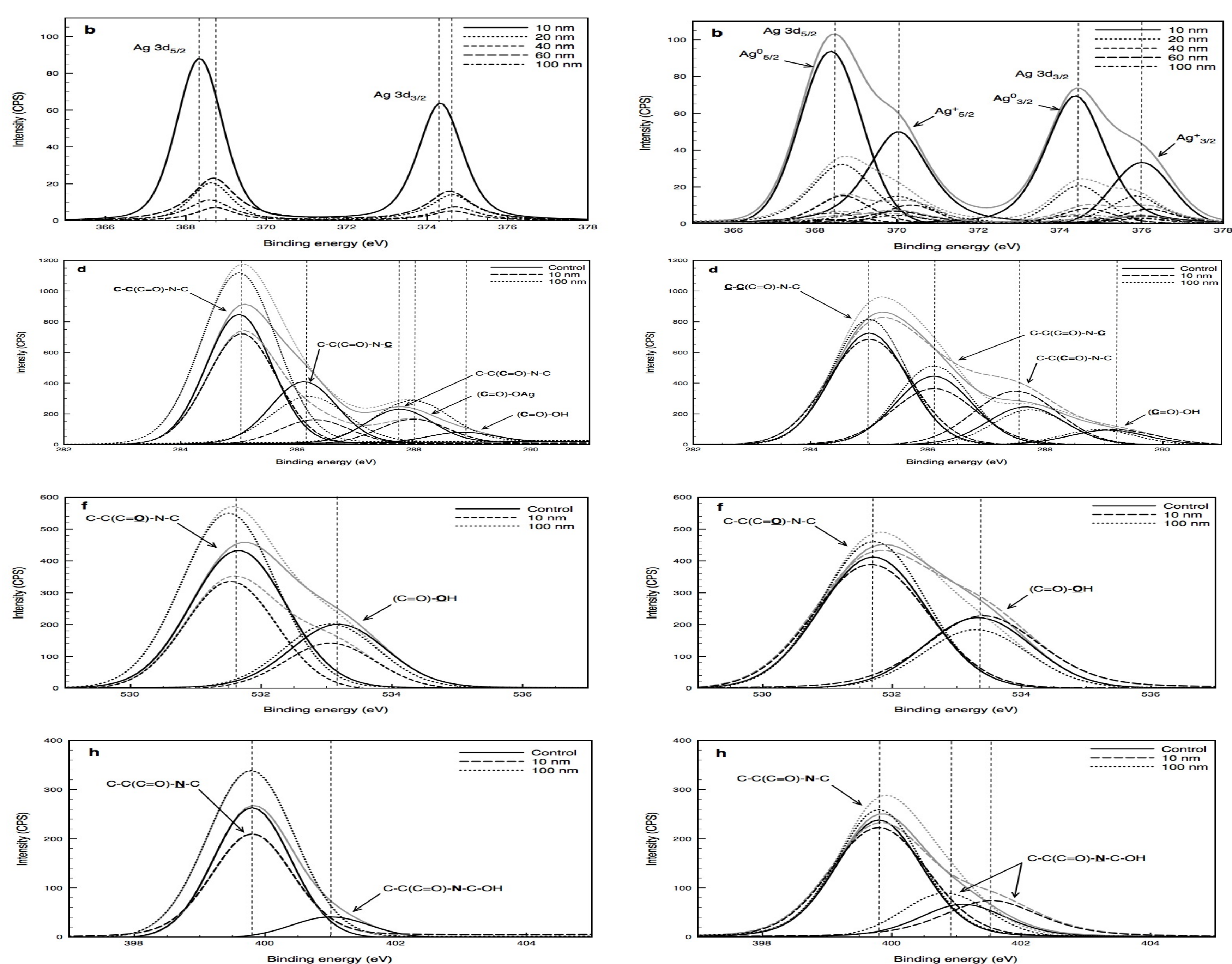

Figure 1 - High-resolution deconvoluted XPS spectra with relative areas of the Ag 3d, C1s, O1s and N1s binding energy regions of plasma treated PA66 fibers surface at day 1 and 30.

After 30 days of aging the nanoparticles-containing fabrics showed a gradual release of $\mathrm{Ag}^{+}$ions from the fabric's surface as confirmed by the presence of two component peaks corresponding to $\mathrm{Ag}^{0}$ and $\mathrm{Ag}^{+}$. The positive shift in the binding energies can be attributed to the polymer-AgO complex. The changes in the N1s e $01 \mathrm{~S}$ core level spectra confirm the occurred chemical changes due to $\mathrm{Ag}^{+}$ions release.

Acknowledgements: This work was funded by Portuguese Foundation for Science and Technology FCT/MCTES (PIDDAC) and co-financed by European funds (FEDER) through the PT2020 program, research project M-ERA-NET/0006/2014 and COMPETE program through FCT within the scope of the project POCI-01-0145-FEDER-007136 and UID/CTM/O
UV-visible spectrophotometric analysis
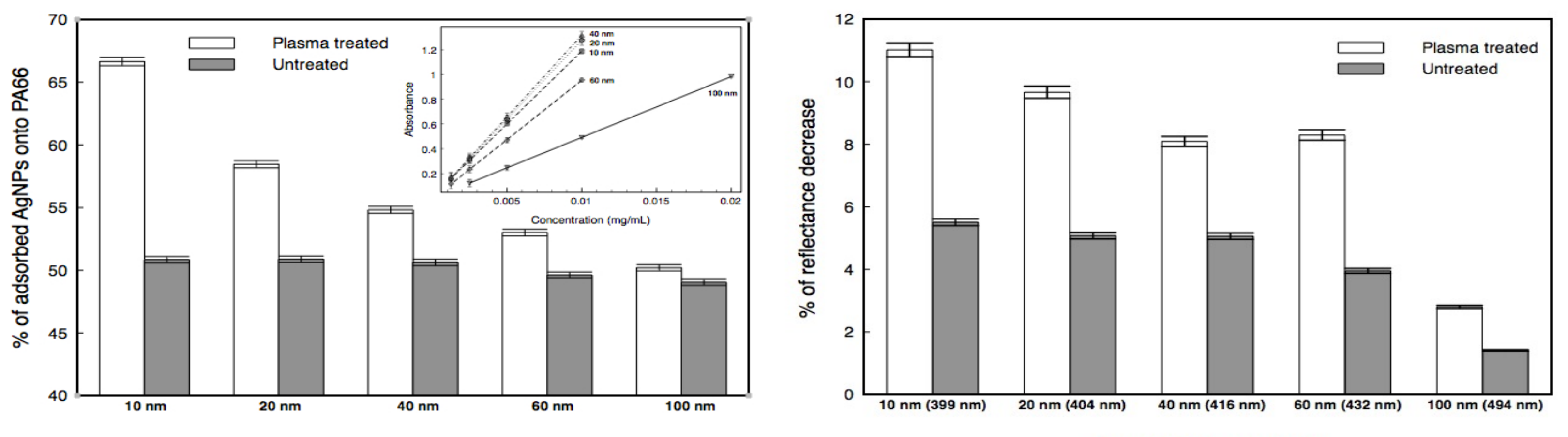

Figure 2 - Percentages of the adsorbed AgNPs (initial concentration of $0.02 \mathrm{mg} / \mathrm{mL}$ ) and decrease in surface reflectance onto the untreated and plasma treated PA66. Inset: Linear fitting of absorption spectra of different size AgNPs vs. concentration; Data are means $\pm S D(n=3)$.

\section{Dynamic contact angle}

The plasma treated PA66 with deposited AgNPs showed, at a different scale, the same size dependent behavior observed on the untreated fabrics. Larger the nanoparticles are, the more pronounced is the wettability effect. After 30 days of aging the smallest nanoparticles deposited on the untreated fabrics showed the highest increase in hydrophobicity.
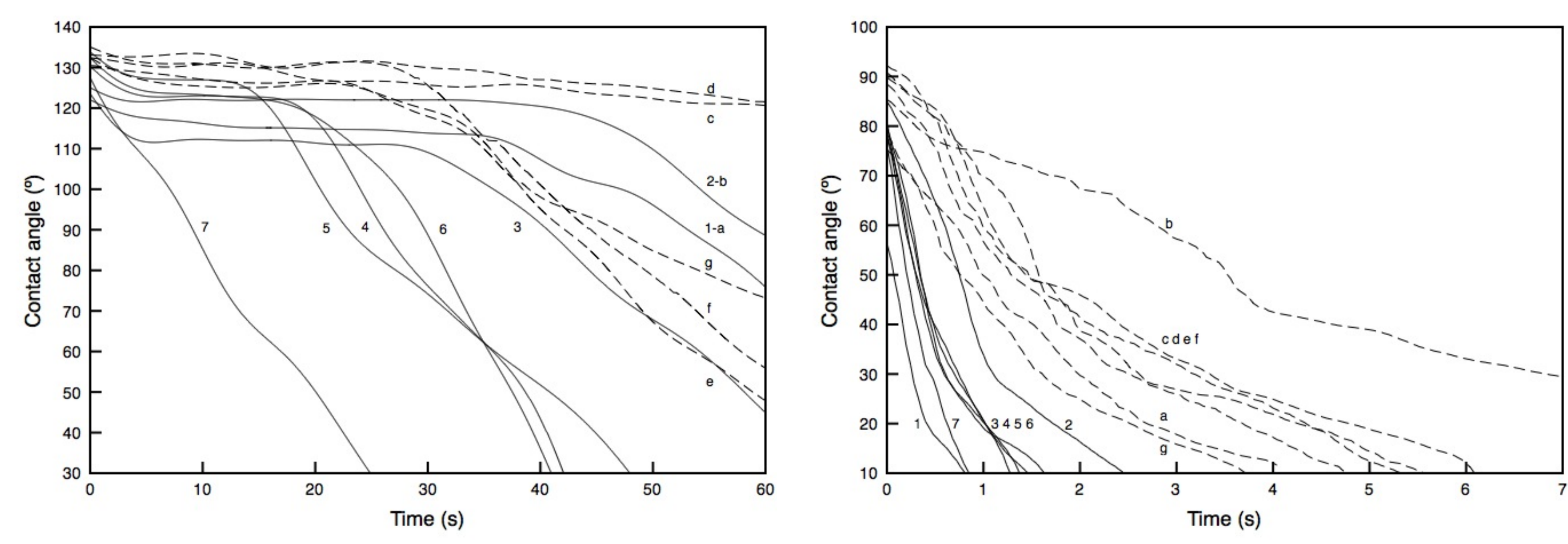

Figure 3 - Dynamic contact angle of untreated and plasma treated polyamide at day 1 (straight lines) and at $\mathrm{t}=30$ days (dashed lines): (1-a) Pristine control; (2-b) Treated control; (3-c) AgNPs $10 \mathrm{~nm}$; (4-d) $20 \mathrm{~nm}$; (5-e) $40 \mathrm{~nm}$; (6-f) $60 \mathrm{~nm}$; (7-g) $100 \mathrm{~nm}$.

\section{Antimicrobial analysis}

The smaller is the diameter of AgNPs, the higher is the antibiotic effect. The observed behavior is attributed to the cell wall composition of grampositive and gram-negative bacteria and to the $\mathrm{Ag}^{+} / \mathrm{Ag}^{0}$ ratio as function of the NPs size. After 30 days of aging, due to the release of silver ions, both the gram-positive and gram-negative strains showed complete inhibition for all the nanoparticle sizes with exception of the $100 \mathrm{~nm}$ NPs.
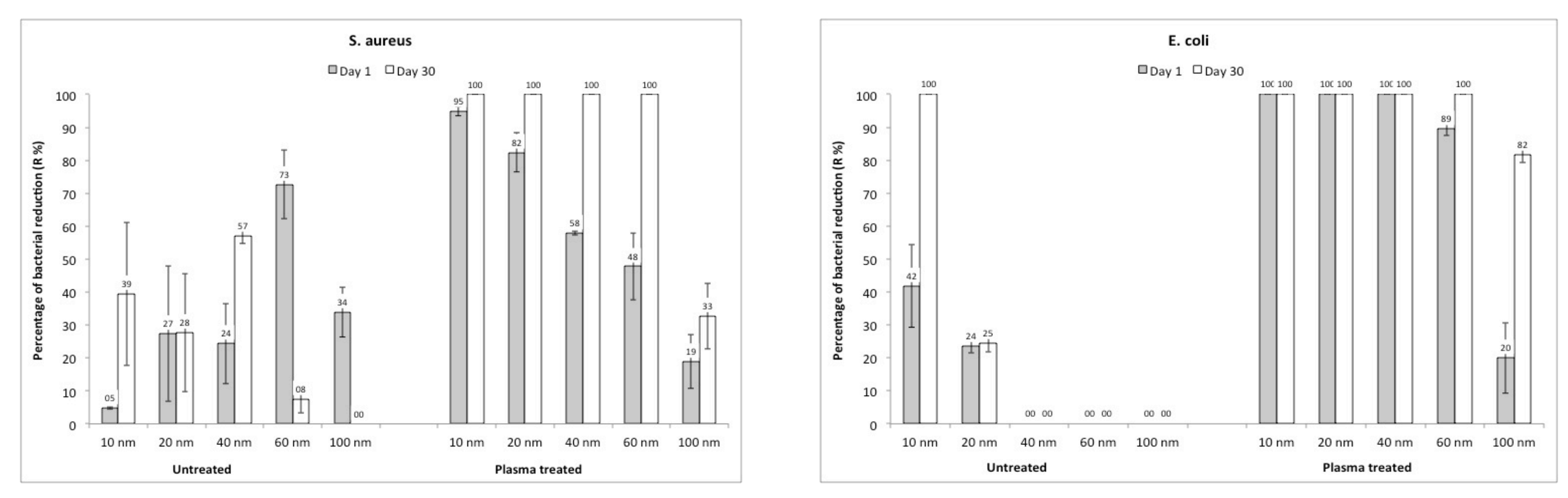

Figure 4 - Percentage of Staphylococcus aureus and Escherichia coli growth reduction respect to the Figure 4 - Percentage of Staphylococcus aureus and Escherichia coli growth reduction respect to the
control fabric of untreated and plasma treated PA66 loaded with different sized AgNPs at day 1 and after 30 days.

\section{Conclusions}

The results of this work display the complexity of the interaction between nanoparticles and polymeric surfaces and suggest that the use of small AgNPs (10-20 nm) as antimicrobial agents can be avoided. 